\title{
NOTA
}

\section{Supervisión de las sociedades de capital tras la crisis financiera}

\section{Marta de Vicente Lama, Horacio D. Molina Sánchez y Jesús N. Ramírez Sobrino'}

\begin{abstract}
En el Coloquio "Hacia una reforma del sistema económico" organizado por la Revista de Fomento Social en Córdoba en mayo de 2011 , se presentó la comunicación que publicamos a continuación, en la que hemos mantenido el estilo más propio de una comunicación oral. Este texto fue objeto de una interesante discusión sobre los aspectos recientemente cambiantes del gobierno corporativo sin descuidar las dimensiones éticas del mismo.
\end{abstract}

Resumen: La profundidad y gravedad de la crisis financiera iniciada en 2007 ha abierto el debate sobre las instituciones que rigen el sistema capitalista. Nuestro trabajo supone una revisión sobre las principales reformas que se han abordado en las mismas, si bien consideramos que gran parte de las soluciones no se encuentran en el rediseño sustancial de éstas, sino en su funcionamiento eficaz. La regulación debe definir nítidamente los principios que se pretenden observar y crear estructuras que garanticen la vigencia de los mismos, abandonando quizás un estilo reglamentista cuyo espíritu, como consecuencia de la evolución acelerada del mundo de los negocios, puede ser fácilmente eludido. Entre estas estructuras consideramos esencial el buen funcionamiento de las entidades porque constituyen la primera "trinchera" en la que se evitan los riesgos. Por otra parte, dado que las entidades gestionan recursos pertenecientes a la sociedad en general y a que, dentro de ella los inversores, premia el comportamiento ético de las empresas, cada vez se está imponiendo una mayor consideración de los "stakeholder" en la toma de decisiones. En nuestro trabajo consideramos que para conseguir la vigencia efectiva del buen gobierno es importante el papel de los consejeros independientes, que en su momento surgieron para representar al capital flotante (accionistas minoritarios en las sociedades cotizadas), pero

1 Profesores de ETEA - Facultad de Ciencias Económicas y Empresariales (Universidad de Córdoba). 
que dada la voluntad de las empresas por comunicar un comportamiento socialmente responsable, se erigen como las personas adecuadas para portar este discurso en el seno del consejo de administración. El reto es conseguir que reúnan las condiciones de competencia e independencia, para lo que proponemos una mayor implicación en su designación de las instituciones encargadas de velar por el funcionamiento ordenado del mercado.

Palabras clave: Capitalismo, Gobierno corporativo, Crisis financiera.

Fecha de recepción: 2 de marzo de 2012.

Fecha de admisión definitiva: 10 de septiembre de 2012 .

\section{Supervision of capital companies after the financial crisis}

Abstract: The depth and severity of the financial crisis that began in 2007 has opened the debate on the institutions that govern the capitalist system. Our work is a review of the major reforms that have been addressed, although we consider that many of the solutions are not in their substantial redesign but in their effective functioning. The regulation should clearly define the principles that are supposed to be observed, and should create structures to guarantee their validity, perhaps abandoning a regimented style whose spirit, as a result of the rapid evolution of the business world, can be easily circumvented. Among these structures, we consider the proper functioning of these entities because this constitutes the first "trench" where these risks can be avoided. On the other hand, given that these entities manage resources that belong to society in general and that, within society, the investors reward the ethical behavior of enterprises, the role of the stakeholder in decision making is becoming more and more evident. Our research considers that the role of independent advisors is important

\section{La supervision des sociétés à capi- taux après la crise financière}

Résumé: L'importance et la gravité de la crise financière qui a débuté en 2007 a ouvert le débat sur les institutions régissant le système capitaliste. Notre travail est une révision des principales réformes abordées dans ces institutions, même si nous considérons qu'une grande partie des solutions ne se trouve pas dans la réforme substantielle de celles-ci, mais dans l'efficacité de leur fonctionnement. La réglementation doit nettement définir les principes à respecter et créer des structures garantissant le fondement de ces principes, en abandonnantéventuellementun style réglementiste dont l'esprit, étant donné les conséquences de l'évolution accéléré du monde des affaires, peut-être facilement évité. Entre ces structures, nous considérons comme essentiel le bon fonctionnement des entités, car elles représentent les premières « tranchées » afin d'éviter les risques. D'autre part, étant donné que ces entités gèrent des ressources appartenant à la société en général, et que, en particulier les investisseurs, récompensent le comportement éthique des entreprises, les stakeholder sont de plus en 
to effectively enforce good governance; they first appeared as representing floating capital (minority shareholders in listed companies), but given the desire of companies to communicate socially responsible behavior, they emerge as the most indicated ones to carry out this discourse within the board. The challenge is to ensure that they meet conditions of competition and independence. We propose greater involvement in the appointment of the institutions responsible for ensuring the orderly functioning of the market.

Key words: Capitalism, Corporate Governance, Financial crisis. plus pris en compte lors de la prise de décisions. Dans notre travail nous considérons que pour réussir à maintenir le fondement effectif du bon gouvernement, il faut tenir compte des conseillers indépendants, dont leur rôle était auparavant de représenter le capital flottant lactionnaires minoritaires dans les sociétés cotisées), mais, qu'étant donné la volonté des entreprises de montrer un comportement socialement responsable, sont devenues les personnes appropriées pour porter ce discours au sein du conseil d'administration. Le défi est de réussir à réunir les conditions de compétence et $d$ 'indépendance; c'est pourquoi nous proposons une plus grande implication dans leur désignation des institutions qui se chargent de veiller sur le bon fonctionnement du marché.

Mots Clés: Capitalisme, Gouvernance d'entreprise, Crise financière.

\section{Introducción}

El capitalismo surge de la particular manera en que se organizaron las empresas para acometer los grandes desafíos que nacieron a comienzos del siglo XVII. La sociedad gremial no podía atender las grandes empresas que surgían del incipiente proceso de globalización que vivió la Humanidad en aquellos siglos y la demanda creció más de lo que los mercaderes europeos consagrados al comercio mundial podían atender. Es en este entorno en el que se comienzan a desarrollar las sociedades de capital, cuya finalidad era la obtención de los recursos necesarios para emprender estos proyectos. El concepto de capitalismo surge de la acumulación de capital como instrumento para el desarrollo (Maddison, 1986); si bien se puede asociar a la forma de organización social, en concreto, la que establece la relación entre los hombres y los bienes materiales utilizados en la producción y que se caracteriza porque admite la propiedad privada de los bienes de producción (Cotta, 1980). Chamberlain (1993) afirma que el capitalismo se fundamenta en promesas, en contratos, gracias a los cuales las sociedades han 
podido abordar proyectos de gran envergadura. En este ensayo nos referimos a la primera acepción de capitalismo.

En el siglo XVII se consolidan las grandes corporaciones como la Compañía Británica de las Indias Orientales o la Compañía Holandesa de las Islas Orientales, entre otras, para dinamizar el comercio de ultramar, fundándose sobre monopolios concedidos por los correspondientes Estados que les permitieron asumir funciones que hoy día les resultan propias como, por ejemplo, la seguridad (Galbraith, 1987). El modelo de captación de recursos resultó un éxito y a partir de entonces sirvió de modelo en el desarrollo de la sociedad industrial en el siglo XIX y en la construcción de las grandes infraestructuras tales como el ferrocarril.

El nacimiento de estas corporaciones fue la respuesta a la captación de capitales necesarios para financiar las empresas. Hasta esa fecha, la financiación procedente de los grandes banqueros holandeses e italianos había dotado a estos proyectos de la liquidez necesaria, sin embargo, el crecimiento de la demanda, unido a las mejoras técnicas que abarataban los costes y reducían los riesgos, permitieron una explosión de los mismos que requería mayor afluencia de capital y la distribución de riesgos entre empresarios y prestamistas.

Las sociedades de capital eran gobernadas por consejos de administración, formados por personas que representaban a los accionistas propietarios. Asimismo, la dimensión de estas sociedades y las necesidades de incorporación de talento específico en el sector de actividad y en las áreas funcionales de la empresa han llevado con el tiempo a la incorporación de un cuerpo de directivos que asumen el poder de gestión en estas corporaciones. La distinción entre propiedad y gestión ha dado lugar a un cuerpo de literatura amplísimo que, con el tiempo, se ha trasladado al derecho positivo de sociedades, bajo la denominación de gobierno de las sociedades.

En lo que llevamos de siglo XXI, la economía ha asistido a dos crisis, la de las empresas tecnológicas y la crisis inmobiliaria y financiera, a un amplio número de escándalos financieros (ENRON, Worldcom, Parmalat, Madoffy, en España, el caso Gescartera, Forum Filatélico, etc.) y todo ello a pesar de una serie de reformas importantes (informe COSO sobre el sistema de control interno, códigos de buen gobierno espoleados ambos por el caso de la Banca Barings a principios de los 90). Tras estos escándalos se han producido modificaciones en la obligatoriedad de los mecanismos de buen gobierno, como la ley Sarbanes-Oxley, la reforma en la normativa bancaria internacional, las reformas en materia de auditoría de cuentas, la reforma de las normas contables o la reforma de los mercados de de- 
rivados. Es decir, tras un escándalo se produce una reacción regulatoria que trata de aprender de la crisis y corregir los posibles excesos; sin embargo, en muchas ocasiones, lo que ha fallado es la vigencia efectiva de la regulación existente. La cuestión resulta inevitable, ènecesita nuestra economía de mercado de más regulación o de mayor eficacia en la existente? Haciendo un símil con el tráfico, ¿ेes preciso reducir la velocidad de circulación o velar porque los mecanismos de control existentes funcionen adecuadamente?, ¿es evitable que se produzca un colapso en un sistema que es naturalmente cíclico? o incluso ¿̇la existencia de un fraude obedece exclusivamente a un fallo regulatorio o a una dejación de las funciones de supervisión y control? Nuestra tesis es que la existencia de colapsos - fraudes es consustancial al sistema, porque la economía es cíclica, porque la codicia humana empuja, en algunos casos, a sortear delictivamente las regulaciones y porque es preciso reconocer una cierta humildad en nuestra capacidad de previsión de cualquier actitud humana.

En gran medida, el sistema capitalista es lo que sean las sociedades de capital que en él operan; nuestro trabajo trata de arrojar luz en lo que, a nuestro juicio, son las principales debilidades en el funcionamiento de estas sociedades y que, - bien merecerían una reforma de la institución jurídica o bien un mayor control por parte de los agentes encargados de desarrollarlo.

Nuestra crítica constructiva al sistema ${ }^{2}$ parte de los fundamentos teóricos de las relaciones surgidas en el seno de las corporaciones, explicados por la teoría de la agencia, el conflicto de intereses al que la regulación se refiere y por unas relaciones en las que existe una asimetría informativa entre agente y principal. El tercer apartado lo dedicamos a presentar los distintos agentes que participan en el buen gobierno de las entidades. En el apartado cuarto mostramos los mecanismos de protección del mercado, introduciendo en el apartado quinto la perspectiva de los "stakeholders"; en el sexto formulamos una propuesta para mejorar la independencia de los consejeros independientes y finalizamos en el séptimo con las conclusiones.

\footnotetext{
${ }^{2}$ Nuestra crítica es constructiva porque no planteamos un modelo alternativo, simplemente pretendemos contribuir a la reflexión sobre los mecanismos cuyo desempeño es preciso potenciar así como señalar alguno de los problemas que se deben resolver desde los terrenos académicos, profesionales e institucionales. La senda no es original pues ya la Comisión Europea plantea la necesidad de someter a examen a las instituciones.
} 


\section{La teoría de la agencia como sustrato conceptual de la rela- ción propietario-directivo en las grandes corporaciones}

La dimensión empresarial aumenta conforme los proyectos a abordar son mayores; por otra parte, el crecimiento empresarial permite diluir los riesgos por lo que, hoy día, la economía de mercado dista mucho de la sociedad gremial con pequeños talleres en el que propiedad y gestión se confundían. Hoy día, una parte importante del sistema económico es atendido por entidades de gran dimensión, no sólo capitalistas, sino también entidades sin ánimo de lucro. Cuando una organización alcanza determinada dimensión, precisa la delegación de funciones y el establecimiento de mecanismos de control de esa delegación. El instrumento teórico que explica estas relaciones es la teoría de la agencia, la cual no da sólo respuesta a las grandes sociedades capitalistas, sino que establece marcos de referencia para organizaciones sin ánimo de lucro.

La relación de agencia se establece a partir de un contrato en el que una o más personas (el principal), encargan a otra persona (el agente) el desarrollo de un servicio en su nombre lo que supone delegar parte de la toma de decisiones en el agente (Jensen y Meckling, 1976: p. 308). La teoría que explica los intereses contrapuestos entre directivos y propietarios se denomina teoría de la agencia. Según la misma, la relación entre agente y principal está sometida a tensiones que es preciso resolver a través de los contratos que se establecen. El directivo, que gestiona el negocio, dispone de mayor cantidad de información que el propietario pudiendo tomar decisiones para maximizar su utilidad y en contra de los intereses de los propietarios. De esta manera, el propietario (principal) tratará de obtener información periódica, información que contrastará con expertos independientes (auditores) y procurará establecer mecanismos para alinear los intereses del directivo (agente) con los suyos propios, como puede ser a través de la formalización de contratos de retribución en los que las variables críticas coincidan con su visión del negocio.

Por otra parte, el agente puede estar tentado de asumir unos riesgos desproporcionados, pues en caso de abordar proyectos rentables, y resultar exitosos, participará del beneficio, pero en caso de resultar desastrosos tan sólo se expondrá a la pérdida de su empleo y, a veces, ni siquiera a eso porque resulte difícil trazar la relación entre las decisiones adoptadas y los resultados de ellas derivados. Este tipo de comportamientos se ve incentivado por mecanismos de retribución basados en la rentabilidad o vinculados al valor de la acción a corto plazo. Existe, en consecuencia, una asimetría en los resultados que incentiva la toma de riesgos por parte de los directivos, especialmente cuando la sociedad dispone de recursos que permitirían asumir los impactos negativos sin ver comprometida su viabilidad. 
En definitiva, entre agente y principal existe una asimetría de información que provoca dos tipos de problemas muy estudiados: la selección adversa y el riesgo moral (Akerlof, 1970). Según el primero, el establecimiento de los contratos entre agente y principal se realizaría en entornos donde la diferencia en el nivel de información entre ambos podría llevar a una toma de decisiones equivocada por cualquiera de las dos partes y se manifestaría, por ejemplo, estableciendo objetivos fácilmente alcanzables por el agente, con el consiguiente desplazamiento de renta del principal al agente. Por su parte, el riesgo moral se produce por la adopción de conductas interesadas por una de las partes en perjuicio de la otra; este problema explicaría conductas en el agente tendentes a abordar proyectos arriesgados sin informar previamente; a utilizar prácticas contables agresivas o directamente a manipular la información financiera. Para evitar estos problemas, el principal puede recurrir a una serie de mecanismos que tratan de reducir o evitar las consecuencias que se derivan de la existencia de asimetrías informativas; por ejemplo, demandando información financiera contrastada adecuadamente por terceros independientes (los auditores de cuentas), participando activamente en la formulación estratégica y en el control de riesgos o incorporando aliados en el control de riesgos, como pueden ser los acreedores financieros.

La cuestión que surge inevitablemente es quién es el principal en la relación de agencia. Desde el punto de vista de la teoría del propietario la respuesta es inmediata, el aportante de recursos que asume el riesgo residual en la organización es el propietario; el corolario es simple: quien asume más riesgo de pérdida económica es el que en última instancia debe tomar las decisiones. El resto de aportantes (los acreedores) tienen un derecho prioritario a recuperar sus aportaciones, por lo que su interés queda protegido por el interés de quien tiene una aportación subordinada.

Desde la perspectiva de la teoría de la entidad, toda la financiación que recibe la empresa son aportaciones de terceros ajenos a la misma; aportaciones con riesgos y beneficios distintos, pero todas ellas suponen obligaciones de la entidad. La entidad se erige como un sujeto independiente de los propietarios. Bajo este enfoque, podríamos considerar que todos los aportantes de recursos participan del devenir de la empresa y deben implicarse en la toma de decisiones. Este enfoque justificaría un mayor nivel de participación en las decisiones de este grupo de "stakeholders" entre los que se encuentran los acreedores financieros y comerciales, los empleados y la Administración. El hecho de no asumir el riesgo último no significa que no asuman riesgos por las decisiones que adopta la empresa; sin embargo, es cierto que su nivel de compromiso financiero es inferior al de los propietarios y que, precisamente, por tener un derecho preferente a los propietarios, tratan de 
obtener mejores condiciones en sus contratos respecto a la entidad, lo que provoca un incremento del riesgo asumido por los propietarios.

Este difícil equilibrio se resuelve actualmente a favor de los propietarios, dado que el incremento de los riesgos que asumirían con una estructura más participativa en la toma de decisiones podría disuadir los procesos de inversión. Sin embargo, dicha preeminencia en el gobierno de la empresa no ha supuesto un abandono de los intereses del resto de aportantes. La solución ha pasado por: a) un mecanismo de mercado en el caso de los acreedores financieros y proveedores; los primeros establecen cláusulas contractuales en los contratos de préstamo o acomodan los tipos de interés al nivel de riesgo; o b) a través de un mecanismo de regulación en el caso de los trabajadores, propiciando una legislación laboral que preserve sus derechos, o en el caso de la Administración tributaria, a través de la legislación fiscal. Actualmente, existe toda una literatura sobre la Responsabilidad Social Corporativa que trata de resaltar el compromiso de la empresa, entre otros, con estos colectivos aportantes de recursos a la entidad.

\section{El esquema del buen gobierno}

Los esquemas de gobierno corporativo surgidos a finales de los 80 , con el informe Cadbury en el Reino Unido y exportados al resto de países con posterioridad, tratan precisamente de facilitar cauces que permitan reducir los problemas derivados de la existencia de asimetrías de información en un marco de relación de agencia. Inicialmente, estos esquemas de buen gobierno fueron contemplados como un derecho "suave"; se concebían como recomendaciones al mercado, que se encargaría de exigir "de facto" su observancia bajo el principio "cumple o explica" al mercado, pero que con el tiempo han sido recepcionados en el seno de las legislaciones con carácter obligatorio. El debate sobre el tipo de regulación necesario en esta materia parece decantarse, tras la Ley Sarbanes-Oxley, por la regulación obligatoria. En esta misma línea, la Comisión Europea (201 la) en el Libro Verde sobre La normativa de gobierno corporativo advierte que las explicaciones ofrecidas por las compañías ante los incumplimientos de los códigos de buen gobierno han sido insatisfactorias y que la supervisión de dichos códigos en algunos países de la Unión Europea ha sido insuficiente.

En los años 80, los avances tecnológicos y de comunicaciones estaban convirtiendo los grandes negocios en mucho más globales y las estructuras de gobierno de las sociedades parecían languidecer frente a una nueva clase, "los directivos"; el funcio- 
namiento de los consejos de administración era insatisfactorio y, en consecuencia, se requería una reforma que propiciase un mayor compromiso en las funciones de gobierno. Las grandes sociedades aglutinan una parte importante del ahorro de las familias; las mejoras tecnológicas y de las comunicaciones han reducido sensiblemente los costes de acceso a la propiedad de las sociedades y los medios de comunicación han familiarizado a los particulares con los mercados de valores, convirtiéndose en una opción frecuente para canalizar su ahorro.

Como consecuencia de lo anterior, las sociedades han incorporado un volumen significativo de propietarios con participaciones reducidas pero que en conjunto representan una porción significativa del capital. Estos recursos aportados por los socios que no son de referencia se conoce como capital flotante y su poder en las decisiones es asumido por los grupos dominantes y por los directivos nombrados por aquéllos. Para evitar la dilución del poder de los accionistas integrados en el capital flotante es por lo que se abordó una reforma del derecho que regula la "vida" de las sociedades de capital, proponiéndose medidas tendentes a mejorar la democracia real en las sociedades a través de medios tecnológicos, especialmente en las juntas generales de accionistas (máximo órgano de gobierno de las mismas) $y$, aparentemente mucho más efectivo, dinamizando las funciones del consejo de administración e incorporando una proporción de consejeros independientes (a los ejecutivos y dominicales -representantes de los grupos dominantes en el accionariado-) equivalente al peso del capital flotante en los recursos propios de la entidad.

El objetivo de los esquemas de gobierno ya no es sólo el control "a posteriori" de la gestión realizada, a través de los informes financieros, sino también del establecimiento de mecanismos de control "a priori" que eviten decisiones con consecuencias fulminantes para las sociedades. Por ese motivo, los mecanismos, además de los clásicos basados en controlar la información vía designación de auditores independientes, se centran en dinamizar los consejos de administración en su funcionamiento y asignarles la función de control "a priori" de la estrategia y supervisión de la gestión de riesgos de la sociedad. La dinamización del consejo pasó por el establecimiento de comités eficaces de menor dimensión y que permitieran preparar las decisiones del consejo de administración, formado por un número más amplio y plural de representantes.

Entre los comités sugeridos por la regulación destacan el comité de auditoría y cumplimiento, cuya función principal es garantizar un alto nivel de calidad en la información periódica preparada por la entidad. Para conseguir este fin, entre los miembros del comité de auditoría no pueden nombrarse consejeros implicados en 
la gestión ordinaria, se debe auxiliar del departamento de auditoría interna, que debería depender de dicho comité y no debería participar en tareas de gestión de riesgos para preservar un adecuado escepticismo profesional, y de los auditores externos a los cuales nombra y con los cuales se reúne periódicamente.

Un segundo comité de gran importancia en el esquema de gobierno corporativo es el comité de nombramiento y retribuciones que trata de preparar los esquemas retributivos de los consejeros y de la alta dirección de la sociedad; de esta forma, se pueden establecer esquemas compensatorios que prioricen la sostenibilidad en el largo plazo. Qué duda cabe que si el accionariado es fundamentalmente especulativo, la actitud de la sociedad tenderá a primar el corto plazo sobre el medio o largo plazo.

Sin embargo, a pesar de estos esquemas de gobierno novedosos, el poder en las sociedades sigue concentrado en los consejeros dominicales y en los ejecutivos. El nombramiento de los consejeros independientes y el mantenimiento de su independencia real es una de las cuestiones cuya solución no parece fácil. En este ensayo tratamos de aportar alguna solución al respecto.

Del conjunto de entidades que operan en la economía, resulta crítico el buen funcionamiento de las denominadas entidades sistémicas, es decir, aquéllas cuyo colapso afectaría seriamente al funcionamiento del mercado. Esta es una de las enseñanzas de la crisis financiera y uno de los debates a posteriori ha sido żcuándo intervenir y cuándo no? Se ha cuestionado que la caída del banco Lehman Brothers agravó la crisis porque introdujo una desconfianza absoluta en el mercado interbancario. Asimismo hemos asistido a un sinfín de intervenciones públicas en bancos y aseguradoras occidentales para evitar su caída (desde la aseguradora estadounidense AIG, al británico Northern Rock y, en España, varias cajas de ahorros requirieron ayuda pública o fueron intervenidas) así como los apoyos financieros temporales concedidos a muchas entidades.

Sin lugar a dudas las instituciones financieras, son entidades vitales en el sistema, el colapso de una entidad financiera puede suponer un terremoto en el mercado, pues son negocios muy endeudados, con una fuerte presencia de acreedores particulares que ante señales de desprotección provocarían una estampida, aceleraría la caída y generaría los riesgos de contraparte en mercados apalancados como el mercado "Over The Counter" de derivados. Por este motivo, la Comisión Europea (2010a) ha emitido un documento que diagnostica los errores que en materia de gobierno de entidades financieras hemos observado durante la crisis. Muchas de estas debilidades también se intuyen en el reciente Libro Verde sobre el gobierno corporativo en la UE (Comisión Europea, 201 la). Entre ellas señala: 
A) Inadecuado conocimiento y escasa dedicación de los consejeros no ejecutivos.

B) Falta de diversidad en la formación de los Consejos.

C) Falta de evaluación del desempeño del Consejo y de la información que llega a él.

D) Débil funcionamiento de los sistemas de control de riesgos en los órganos de gobierno (desconocimiento de los sistemas de riesgo y de la gestión del riesgo, inadecuada consideración de la función o la recepción tardía de la información).

E) Falta de independencia en los mecanismos de control de riesgos.

F) Estructuras de remuneración inadecuadas que dan lugar a la asunción de riesgos y a una visión cortoplacista.

G) Escasa implicación de los inversores institucionales en los consejos de las entidades financieras (debido entre otros motivos a que su modelo de negocio se centra en la negociación de carteras).

H) Escasa implicación de los inversores individuales por los elevados costes.

I) Conflictos de interés de los gestores de activos financieros respecto a las entidades financieras.

J) Escasa comunicación entre los miembros del Consejo y los supervisores en cuanto al buen gobierno de las entidades.

El documento de la Comisión (Comisión Europea, 2010a) advierte que estos errores están en contradicción con los principios establecidos en los esquemas de gobierno, ¿̇qué es lo que ha fallado?; ¿ los principios son demasiado laxos y permisivos, siendo posible ofrecer una imagen de cumplimiento sólo en apariencia? - ìha fallado la vigencia efectiva de los mismos, porque ni los accionistas ni los supervisores aplicaron la debida diligencia?

Las respuestas desde la Unión Europea han sido la emisión de recomendaciones y medidas legislativas en la directiva sobre requisitos de capital referentes a la política de remuneración.

Los documentos anteriores (Comisión Europea 2010a y 201 la) señalan un conjunto de buenas prácticas que, implican una reforma del sistema (ver tablas 1-3). 


\section{TABLA I. Propuestas referentes al Consejo de Administración y los consejeros}

\begin{tabular}{|l|}
\hline I. SOBRE EL CONSEJO DE ADMINISTRACIÓN \\
\hline a) Limitar las reelecciones de los consejeros no ejecutivos así como exigir una dedicación cuantificada en \\
un mínimo de días al año (2.2.1). \\
\hline b) Establecer políticas para garantizar la competencia y la independencia. Entre ellas, requerir formación \\
continua a medida (2.2.2). \\
\hline c) Procurar un grado elevado de diversidad entre los miembros del Consejo (2.2.3). \\
\hline d) Facilitar asesoramiento externo a los miembros del Consejo (2.2.4). \\
\hline e) Mejorar la evaluación del funcionamiento del Consejo incluso con el empleo de externos para tal \\
misión (2.2.5). \\
\hline f) Definir claramente las responsabilidades en materia de la definición e implantación de la estrategia, \\
así como el control de riesgos. En las instituciones financieras, los consejeros independientes deben \\
velar también por los intereses de los depositarios. La exigencia de responsabilidades es una materia \\
controvertida pues es preciso buscar equilibrios que eviten las malas prácticas pero que el nivel de \\
exigencia no disuada de contar con consejeros competentes (2.2.6). \\
\hline g) El Consejo debe aprobar el apetito de riesgo de la institución financiera y supervisar el alineamiento \\
de la estrategia corporativa con dicho perfil de riesgo deseado. La alta dirección debe facilitar la toma de \\
conciencia para la decisión por parte del Consejo (2.2.7). \\
\hline h) Disponer de canales de comunicación directos del Consejo de Administración con el Director de \\
Riesgos (2.2.7). \\
\hline $\begin{array}{l}\text { i) Ofrecer al mercado información sobre la política de riesgo, como indicador de la solidez del marco de } \\
\text { gestión de riesgo (2.2.7). }\end{array}$ \\
\hline j) Crear un Comité de riesgos formado por consejeros independientes. Asimismo, el Presidente el \\
Comité de Auditoría debe participar en el Comité de Riesgos y viceversa (2.2.7). \\
\hline k) Establecer políticas retributivas basadas en el crecimiento sostenible a largo plazo, considerando las \\
implicaciones sobre el riesgo (2.2.8). \\
\hline I) Diálogo con los supervisores (2.2.9). \\
\hline
\end{tabular}

Fuente: Elaboración propia a partir de Comisión Europea (2010a).

\section{TABLA 2. Propuestas referentes a la gestión de riesgos}

II. SOBRE LA GESTIÓN DE RIESGOS

a) Definición, validación y revelación de la política de riesgos (apetito, perfil y parámetros para medirlo) (3.2.1).

b) Establecer políticas para garantizar la independencia del Director de Riesgos, equiparándolo orgánicamente al Director Financiero (3.2.2).

c) El personal de riesgos debe tener conexión a través del Director de Riesgos, pero también a nivel operativo con las diferentes unidades (3.2.3).

d) Dedicar recursos a construir una cultura de gestión del riesgo (3.2.4).

e) Establecer sistemas de retribución que velen por el crecimiento a largo plazo en lugar de la toma de riesgos cortoplacista (3.2.5).

Fuente: Elaboración propia a partir de Comisión Europea (2010a). 
TABLA 3. Propuestas referentes a los accionistas

\section{SOBRE LOS ACCIONISTAS}

a) Propiciar la adopción de códigos de inversión, bajo el principio "cumple o explica". La revelación del voto de los inversores es clarificador sobre la implicación de los inversores y en cómo se resuelven los conflictos de interés (4.2.1).

b) Incrementar la transparencia de los mandatos que realizan los inversores institucionales a sus gestores de activos (4.2.2).

c) Creación de foros para buscar la cooperación entre accionistas y la comunicación de información, de manera que se reduzcan los costes de una implicación permanente (4.2.3).

d) Establecer políticas de identificación, gestión y comunicación de los conflictos de interés (4.2.4).

e) Revelar más información y más comprensible sobre la compañía y los riesgos, procurando informar de las reuniones bilaterales con accionistas (4.2.5)

f) Mejorar la claridad de las actuaciones concertadas (4.2.6)

g) Mejorar la participación de los accionistas en la toma de decisiones (4.2.7).

Fuente: Elaboración propia a partir de Comisión Europea (2010a).

Este panel de medidas propuestas desde la Comisión Europea va más dirigido a reforzar la vigencia efectiva de los principios de gobierno corporativo que, propiamente, a una reforma sustancial del esquema de gobierno. La estrategia pasa, por tanto, en ganar vigencia efectiva a través de una regulación basada en grandes principios, con buenas prácticas que los desarrollen y una actividad supervisora que vele por el seguimiento de los mismos, frente a una concepción reglamentista, que establezca "a priori" unos mecanismos que pueden burocratizar la actividad sin añadir calidad a la toma de decisiones. El dilema es una regulación detallada "a priori" o una regulación sobre principios y buenas prácticas supervisadas en función de las peculiaridades del negocio o de la dimensión empresarial.

La siguiente cuestión es quién debe supervisar, ¿̇un órgano externo o el propio mercado? Hasta el momento, la concepción ha sido que el propio mercado fuese el que premiase o castigase la falta de transparencia o las políticas inadecuadas de gobierno; sin embargo, la escasa vigencia efectiva de los códigos de buen gobierno ha dado lugar a que, en las entidades sistémicas, los supervisores se planteen que deben tener una implicación más continua. 


\section{Mecanismos de protección del mercado}

Adicionalmente a los mecanismos de buen gobierno, diseñados para proteger los intereses de los accionistas, las entidades que cotizan en mercados de valores se someten a la vigilancia de los supervisores del mercado de valores (en España, la Comisión Nacional del Mercado de Valores), que protege a los posibles inversores a través de la exigencia de una política de transparencia de las entidades cotizadas.

Las entidades financieras se someten al supervisor bancario que vela por los intereses de la economía en general, por ser entidades críticas para garantizar el flujo financiero, y por la de los depositantes en particular. De igual manera, las entidades de seguros se someten a la Dirección General de Seguros quien garantiza la viabilidad de este tipo de entidades que asegura el ahorro a largo plazo que canalizan estas entidades.

Una cuestión que se ha planteado al hilo de algunos escándalos en España (caso Forum Filatélico, AFINSA y recientemente la suspensión de pagos del Grupo RUMASA, tras una agresiva campaña de marketing en la que se comercializaban productos financieros al público en general, ofreciendo elevadas rentabilidades) es la responsabilidad subsidiaria del Estado ante este tipo de actividades que toman ahorro de particulares con mínimas o nulas garantías. Formalmente, no estaban sometidas a supervisión alguna, pero claramente estábamos en presencia de cómo la letra de la ley era superada ampliamente por unos hechos que hubiesen requerido una acción legislativa correctora.

La protección del mercado no pretende sustituir el criterio de los inversores a la hora de decidir qué rentabilidades se deben obtener y qué riesgos se pueden asumir; su misión consiste en garantizar que los inversores disponen de toda la información existente en el mercado, tratando de preservar la igualdad de oportunidades de todos los "jugadores del tablero" (regulación para prevenir la información privilegiada), así como toda la información sobre los riesgos y estrategias de los negocios. En cualquier caso, el mínimo de información a comunicar viene determinado por las normas contables, ahora en proceso de armonización internacional. Por otra parte, de manera voluntaria, las páginas "webs" de las compañías cada vez recogen más información sobre los negocios, estrategias, presentación de resultados a analistas, seguimiento e informes de los mismos. Este camino es, a nuestro juicio, el adecuado: preservar la igualdad de oportunidades y propiciar una política de máxima transparencia. 
La preparación de la información financiera recoge, por un lado, la información histórica a partir de la cual se realizan las proyecciones de los flujos de efectivo y revelándolos según los diversos segmentos de operación de los grupos empresariales. A finales de los 90, con motivo del crecimiento del sector tecnológico se puso de manifiesto la importancia que tenían ciertos activos no reconocidos en los balances, dado que carecían de una medida fiable y no era prudente reconocerlos, pero que, sin embargo, eran determinantes para explicar los flujos de efectivo futuros del negocio, nos referimos a los activos intangibles (el conocimiento, la marca, la organización, la calidad de los recursos humanos). Posteriormente, fue la revelación de los valores razonables de las carteras mantenidas para negociar o el valor razonable de los inmuebles de inversión. El dilema era comunicar esta información y de esa manera garantizar la igualdad de oportunidades entre los diversos agentes participantes o ser prudente y no revelar la información. En el mercado, basándose en la información más relevante se podían obtener plusvalías porque parte del mercado no disponía de esa información; sin embargo, la presentación paulatina del valor razonable de determinados elementos contables suponía ofrecer una información más relevante pero menos fiable e introducía más riesgo en la valoración de las compañías. Y, he aquí, que al final la culpa de la crisis parece que fue esta valoración a valor razonable. $Y$ eso con independencia de ser bien sabido la menor fiabilidad de dicha información. El mensajero no puede ser el responsable.

Fruto de esta insatisfacción, el G-20 (2009), en la reunión de Pittsburg, exhortó a los organismos reguladores contables de Estados Unidos (Financial Accounting Standards Board -FASB-) e internacional (International Accounting Standards Board -IASB-) a que redoblasen sus esfuerzos de convergencia. Recientemente, se han aprobado normas sobre consolidación (y evitar de esta manera la ocultación de pérdidas o deuda), valor razonable e instrumentos financieros. Asimismo, en la agenda existe una importante reforma en materia de arrendamientos que pretende incorporar al balance la información sobre los compromisos adquiridos en estos contratos y que los analistas incorporan tras un proceso de ajuste de la información contenida en la memoria.

Otro campo de batalla en materia de información ha sido la dotación de pérdidas de crédito. Actualmente, el sistema está siendo revisado pues ha generado una gran insatisfacción. Los créditos de larga duración es previsible que tengan que sufrir tasas de morosidad superiores a las que experimentan en el momento de la concesión, por lo que desde el principio debe registrarse el crédito considerando la rentabilidad real, descontando la parte de los flujos contractuales que es probable que no se cobren. Evidentemente, la esperanza de cobro es positiva y el tipo de 
interés permite ajustar dichas pérdidas para que la rentabilidad sea razonable $y$, en caso de no poder obtenerse, rechazar la operación. Este razonamiento introduce un elemento novedoso en la contabilidad que es informar sobre hechos futuros, una línea peligrosa, que se atraviesa en sentido simétrico cuando se valora a valor razonable sin una referencia de transacción en el mercado (a través de descuento de flujos de efectivo) o asimétricamente, con prudencia, cuando existe una pérdida potencial.

No obstante, esta línea de argumentación es consistente conceptualmente con la transparencia, la que no lo es, es la de exigir a la información financiera unos efectos sobre la estabilidad de las entidades; esta misión le corresponde al regulador bancario con los requisitos mínimos de capital. De hecho, el presidente del IASB, Sir David Tweedie (2010), propone la elaboración de un documento de pérdidas y ganancias específico para los fines regulatorios, manteniendo la tradicional cuenta de pérdidas y ganancias bajo criterios de transparencia. Si las entidades financieras presentan problemas de solvencia no es por las normas contables, salvo que técnicamente no reflejen la imagen fiel, sino porque la regulación bancaria es arriesgada. En este sentido, no es inconveniente exigir requisitos de capital variables en función de la fase del ciclo.

El dilema es el siguiente, ¿debe la información ser fidedigna o debe favorecer el buen funcionamiento del sistema aun a costa de generar unas rentas a los "insiders"?; ¿ ¿a quién corresponde velar por el buen funcionamiento del sistema a los supervisores o al regulador contable?

Las crisis financieras terminan suponiendo una crisis del crédito de los agentes que intervienen en el sistema financiero; se desconfía de su capacidad para atender sus compromisos, lo cual conduce a que se retraigan las operaciones crediticias. Esta situación ha puesto ante el foco a unas instituciones un tanto desconocidas, porque su peso es menos relevante, en periodos de bonanza. Se trata de las agencias de calificación crediticia (agencias de "rating"). Estas agencias suministran al mercado, tras exhaustivos análisis de las compañías y Estados que estudian, una recomendación de la calidad crediticia de sus emisiones. En función de dichas calificaciones los mercados exigen mayores primas de riesgo, en definitiva, intereses más elevados, a las compañías o Estados con peores perspectivas crediticias. El peso de estas calificaciones es muy importante y sin embargo el riesgo de crédito es muy difícil de valorar porque no es un suceso observable frecuentemente en una compañía; las entidades quiebran una vez, por lo que las predicciones (o valoraciones de salud financiera) parten de la hipótesis de que dos compañías, con datos financieros similares, se comportarán de manera similar y si un grupo 
de ellas quebró en el pasado, las probabilidades de que la observada quiebre es también elevada. Evidentemente en un modelo de predicción es difícil incorporar variables que, en nuestra opinión, son importantes para la gestión de un negocio y la superación de una crisis en el mismo y además son únicos (difícilmente reproducibles en otros negocios). Este el el caso, por ejemplo, de nivel de competencia del equipo directivo, de los activos no reconocidos como intangibles o del contexto económico y las causas que lo determinan. En este sentido, un trabajo de revisión de la literatura sobre predicción de la quiebra empresarial, realizado por Balcaen y Ooghe (2006), pone de manifiesto que los modelos clásicos de predicción, entre otras debilidades, no obtienen buenos resultados de predicción en muestras con características diferentes a las que se emplearon para construir el modelo.

Por otra parte, la financiación de las agencias crediticias responde a un modelo de mercado y procede de las propias entidades analizadas, siendo además un mercado muy concentrado. Este modelo es similar al que rige en el mercado de auditoría, igualmente concentrado, y no hay que ocultar que tiene sus detractores (Sikka, 2009).

Recientemente, la Comisión Europea (2011c) ha emitido una propuesta de Reglamento para reformar la regulación sobre las agencias crediticias sobre cuatro pilares: a) reducir la dependencia de sus calificaciones y del escaso número de agencias; b) mejorar su independencia; c) obligarles a ser más transparentes sobre los métodos y evaluaciones utilizados para la obtención de la calificación crediticia así como a realizar con más frecuencia sus evaluaciones en determinadas entidades y d) mejorar las vías de reclamación de los damnificados.

Finalizamos este apartado de protección del mercado con un breve comentario sobre los auditores. Tradicionalmente la auditoría protegía los intereses de los accionistas y hubiese tenido sentido tratarlo en el tercer apartado, sin embargo, la profesión de auditor se ha consagrado como una actividad de interés público y eso le acerca más a ser un mecanismo de protección de mercado. La calidad de la información exige un contraste independiente de la sociedad que la emite y este es el rol del auditor. Para desarrollar su trabajo, el auditor debe comprender el negocio del cliente, así como los riesgos que enfrenta, por lo que fruto del mismo se derivaría una información muy relevante para los comités de auditoría y los supervisores; pero el producto principal es un informe en el que se detallen aquellas salvedades significativas que deterioran la calidad de la información. Los controles que establece la regulación para preservar su independencia son "a priori", estableciendo salvaguardas que impedirían la aceptación de un encargo de auditoría, o bien "a posteriori", a través de la supervisión de las firmas. 
El control "a priori" de la independencia del auditor incluye un severo régimen de incompatibilidades personales y de prestación de servicios en los que pudiese generarse un conflicto de intereses, pero ello no previene de un riesgo sobre la independencia que es el hecho de percibir los honorarios de la firma auditada, quien además es quien decide su contratación. Para evitar estos riesgos a la independencia, el Libro verde de reforma de la auditoría, emitido por la Comisión Europea (2010b), va más allá de un nombramiento por parte del comité de auditoría y plantea la posibilidad de un nombramiento por parte de una autoridad reguladora, o que al menos tenga lugar en las entidades sistémicas. Este planteamiento es consistente con la consideración de actividad de interés público, incorporando como destinatarios del informe no sólo a los accionistas contratantes, sino a cualquier tercero que hubiese confiado en él. La prevención de conflictos de interés entre la sociedad y los terceros es la causa por la que se plantea el nombramiento por parte de una autoridad reguladora, si bien se reconoce que el incremento de la burocracia podría desaconsejar la medida ${ }^{3}$.

Por otra parte, el Libro Verde solicita la opinión de los "stakeholders" sobre la idoneidad de la rotación obligatoria del socio y de la empresa de auditoría firmante del informe (solución existente en España durante un tiempo). En este caso, la cuestión a decidir es si la pérdida de eficacia en la realización del trabajo, fruto del alisamiento de los costes de conocimiento inicial de la entidad auditada en un periodo más amplio, compensa la posible pérdida de independencia procedente de la familiaridad de las relaciones.

Una segunda cuestión importante en la regulación del mercado de auditoría es el control "a posteriori" sobre la actividad realizada por los auditores. El organismo que realiza dicha supervisión debe ser público, aunque colabore con instituciones privadas; la cuestión radica en la supervisión de las redes internacionales que realizan trabajos de auditoría sobre compañías transnacionales.

Tras la recepción de las cartas de comentario a dicho Libro Verde la Comisión extrae las siguientes conclusiones para las entidades de interés público (entidades con relevancia pública especial por su dimensión o porque disponen de un amplio

${ }^{3}$ Sikka (2009) propone un modelo diferente al de mercado para las entidades financieras; en concreto propone un cuerpo de inspección que permitiría a los supervisores conocer mejor la situación de las entidades. En España, la inspección del Banco de España desarrolla este papel y no por ello se ha conseguido evitar algunos casos de dificultades financieras severas. El mismo autor indica que la crisis financiera ha puesto de manifiesto un conocimiento insuficiente de los riesgos del sector financiero por parte de los auditores. 
número de "stakeholders") y, las cuales forman la base del futuro Reglamento que se encuentra en fase de propuesta (Comisión Europea, 201 ld): a) existe una diferencia de expectativas entre lo que los interesados esperan y lo que los auditores realmente hacen; b) la independencia no está garantizada si la auditoría representa un servicio comercial más; la inexistencia de licitaciones periódicas y una insuficiente rotación dañan la independencia del auditor que es una característica básica y c) una elevadísima concentración del mercado en las Big 4 (las cuatro firmas más importantes: Deloitte, Pricewaterhousecoopers, Ernst\&Young y KPMG).

La solución legislativa a estos problemas en el mercado de la auditoría de las entidades de interés público pasa por: a) clarificar el alcance de la auditoría legal y mejorar la comunicación con los usuarios; b) prohibición de prestar servicios adicionales a las entidades auditadas así como unas normas más estrictas sobre designación y rotación de auditores; c) prohibir las cláusulas contractuales que limitan la selección de auditores, creando un certificado de calidad de firmas de auditoría; d) limitar las restricciones que afectan a la propiedad de las firmas y e) fortalecer las entidades supervisoras nacionales y coordinar los esfuerzos a nivel europeo.

\section{La ética en el gobierno de las entidades}

Según el IFAC (2012), los inversores necesitan información sobre el desempeño ambiental, social y de gobernanza para conocer mejor la organización y cómo estos aspectos impactan en el desempeño a largo plazo, fundamentalmente reduciendo los riesgos en dicho horizonte. Asimismo, señala que juegan un papel esencial en el desarrollo de esta perspectiva con la limitación de la visión a corto plazo y la pasividad de los propios inversores. En concreto, el IFAC (2012) señala cuatro factores que justifican esta tendencia: a) el número de inversores que han firmado los Principios de Inversión Responsable de Naciones Unidas; b) el número creciente de propuestas de accionistas que comprenden resoluciones ambientales, sociales y de gobierno; c) las diversas encuestas que apuntan que la integración de esta perspectiva en el proceso de inversión maximiza el interés de los beneficiarios a largo plazo y que un buen tono de gobierno y prácticas sostenibles contribuyen a la creación de valor a largo plazo a los accionistas y d) un cuerpo de investigación que muestra cómo los inversores cuando incorporan en sus decisiones de inversión la información sobre sostenibilidad, de carácter social y relativa al gobierno mejoran su nivel de supervisión. 
La demanda de un tono ético más elevado a las empresas encuentra diversas explicaciones, unas de carácter estructural como puede ser una mayor exigencia del mercado sobre la forma en que la empresa fabrica sus productos o presta sus servicios y otras de carácter coyuntural, esto es, la ética como factor que puede mitigar la concurrencia de fraudes empresariales o incluso como amortiguador de las crisis económicas al propiciar comportamientos menos especulativos (Comisión Europea, 2011b).

El motivo más estructural al comportamiento ético de la empresa se encuentra en la percepción de la sociedad (que al fin y al cabo es el mercado de la empresa) sobre cómo una entidad toma sus decisiones (por ejemplo, de carácter social tales como las subcontratas en condiciones laborales adecuadas), cómo fabrica sus productos (por ejemplo, si utiliza procedimientos sostenibles medioambientalmente) y cómo se relaciona con su entorno y procura su desarrollo. Los consumidores conforme disponen de un poder adquisitivo más elevado no sólo deciden en base al binomio precio-calidad sino que sus decisiones de compra consideran esos otros factores mencionados; y en una sociedad desarrollada la proporción del mercado con esta sensibilidad más amplia es más elevada. La sociedad se ha vuelto extremadamente crítica con los mensajes propagandísticos de algunas empresas, aumentando el gap de expectativas entre lo que espera y lo que hacen las empresas (Comisión Europea, 2011b).

Como factor coyuntural, suponiendo que se ha seguido la legislación escrupulosamente, lo que suele esconder esta denuncia es el exceso de riesgo asumido por la empresa. En este punto es conveniente distinguir la asunción de riesgos que es propia de la actividad empresarial, bajo la regla de "a mayor riesgo mayor rentabilidad exigida al proyecto", de asumir riesgos no conocidos o no deseados por los propietarios o asumir riesgos que en el largo plazo perjudicarán a la sociedad en general (por ejemplo, actividades contaminantes aunque legalmente admitidas). En periodos expansivos, los inversores demandan niveles mayores de rentabilidad $y$, en ese contexto, qué administradores o qué directivos son capaces de limitar la "fiesta" sin que los inversores les den la espalda o sin que los directivos sean fulminados por un bajo desempeño.

La explicación de las relaciones en la empresa desde la teoría de los "stakeholders" encuentra su fundamento en la teoría del bien común (Argandoña, 1998). Las entidades no sólo gestionan los recursos que controlan como propietarias, sino que también cogestionan con el resto de la Humanidad una serie de bienes públicos. El disfrute de los mismos debe procurar su preservación para el resto de ciudadanos y entidades así como para las generaciones futuras. En otro caso, 
se está produciendo una apropiación económica de dichos bienes públicos. Sin adoptar posturas maximalistas, el desarrollo de la actividad de las entidades y de los ciudadanos debe tender a preservar el medioambiente y a reparar los daños que se deriven de la actividad. En el caso de que no exista una tecnología adecuada para procurar dicha restauración, el dilema reside en determinar si es admisible afrontar el desgaste, teniendo en cuenta, en el otro lado de la balanza, el desarrollo de la sociedad que se consigue con dicha actividad. Evidentemente, este cálculo de oportunidad debe considerar los intereses de las generaciones futuras y transitar más allá de una visión cortoplacista.

De esta manera, el concepto de riesgo debe ir más allá de la entidad, no pudiéndose reducir exclusivamente a los propietarios de la misma. La realización de actividades tiene impacto en el medioambiente y el gobierno de las sociedades debería incorporar una visión respetuosa con estos recursos. A nivel global, las declaraciones del G-20 consagran una salida de la crisis que considere un crecimiento sostenible bajo el principio de "responsabilidades comunes pero diferenciadas" (G-20, 2009a y G-20, 2010).

La preocupación medioambiental también ha sido percibida por las corporaciones y paulatinamente han comenzado a divulgar, de manera voluntaria, información sobre los impactos medioambientales de sus actividades. Al mismo tiempo, los reguladores, han exigido revelar el impacto económico que se puede derivar de las actuaciones medioambientales.

Asimismo, los gobiernos se han comprometido a reducir las emisiones de gases efecto invernadero, creándose un mercado de cuotas de contaminación, de tal manera que las empresas tienen un límite de emisiones que, caso de ser superadas, les obligarían a comprar derechos de emisión. Por su parte, con la finalidad de incentivar las actividades no contaminantes, las empresas pueden obtener derechos de emisión de los gobiernos si desarrollan actividades "limpias". En definitiva, se ha creado un mecanismo de mercado para limitar la contaminación, bajo el principio de "quien contamina, paga" y que, asimismo, permite redistribuir los beneficios de la actividad empresarial entre todos los países que sufren el deterioro de un bien común como es el medioambiente.

Una dimensión ética de la empresa debería considerar no sólo cuántos resultados obtiene la empresa sino cómo toma sus decisiones; la cuestión es j̇cómo hacer posible el concurso de la ética en el mundo de los negocios?

La estrategia de la Unión Europea pasa por un mayor nivel de concienciación sobre las ventajas de la responsabilidad social (a nivel de promoción de las inicia- 
tivas empresariales en esta materia; apoyando las iniciativas de autorregulación, impulsando los proyectos supranacionales de mejores prácticas; impulsando la educación, entrenamiento e investigación, de la contratación pública; promoviendo la concienciación de los mercados y de los consumidores) y de información pública sobre las actividades de responsabilidad social emprendidas, idealmente en el marco de la información anual (Comisión Europea, 2011 b).

En nuestra opinión, la información sobre responsabilidad social es una señal que la empresa manda a la sociedad (sus "stakeholders" en definitiva) para indicarle que las decisiones responden a una determinada ética que no sólo considera el resultado financiero, sino que también incorpora una determinada forma de "hacer las cosas". Pero quizás no basta con sólo informar, es preciso integrar la visión responsable en los procesos de decisión tratando de evitar lo que el IFAC (2012) denomina como actitud de mero cumplimiento. Los guardianes de esta ética deberían ser, en nuestra opinión, los consejeros independientes, figura que surgió para defender un interés general (el de los accionistas minoritarios), pero que en una visión de la empresa más allá de sus "shareholders" (accionistas), una visión que considere su responsabilidad con los "stakeholders", puede ser encargada a dicho grupo de consejeros, con la finalidad de ser representantes de los intereses más amplios de la sociedad en general.

La ética en los negocios es un mensaje que, si cabe, es más importante en las entidades sin ánimo de lucro; entidades que nacen para desarrollar su actividad con un estilo más allá de los resultados de la actividad. Los excedentes no dejan de ser un indicador de la sostenibilidad en el largo plazo; una forma de controlar que la actividad desplegada recibe la financiación necesaria para ser mantenida de manera continuada en el tiempo, no generando riesgos excesivos a los "stakeholders" de la entidad sin ánimo de lucro; en definitiva los resultados son un medio que no el fin para el que nacieron, el estilo es especialmente importante.

\section{Los consejeros independientes y la difícil cuestión de su independencia}

De las diversas medidas que introdujeron los códigos de buen gobierno, una de las más destacadas, en nuestra opinión, fue la incorporación de la figura del consejero independiente y los motivos por los que se hacía: en representación del capital flotante (Código Olivencia, 1998). 
Por otra parte, parece razonable que en un marco legal que se define como economía social de mercado, las empresas deban concebirse bajo la teoría de los "stakeholders" y deban tender a considerar el impacto que sus actuaciones tienen en su entorno. La adopción efectiva de una actitud socialmente responsable debe formar parte de la cultura de la empresa y para ello, debe emanar desde su máximo órgano de gobierno. Los consejeros independientes pueden ser los encargados en dicho órgano de velar porque las decisiones que se adoptan conjuguen criterios de sostenibilidad y responsabilidad, tal y como la sociedad civil demanda. Por tanto, los consejeros trascienden su función a ser meramente depositarios de la confianza del capital flotante para pasar a ser los encargados de velar por el interés público en la sociedad.

Sin embargo, a pesar de la bondad de la propuesta, su vigencia efectiva choca, como ya hemos expresado, con la difícil cuestión de quién los nombra y la independencia que realmente poseen por la procedencia del nombramiento (la comisión de nombramientos donde se dan cita consejeros dominicales e independientes).

En este sentido, el consejero independiente no sólo debe reunir las características profesionales que lo hagan apto para contribuir al negocio, sino que debe disfrutar de independencia respecto a los consejeros dominicales y ejecutivos. La garantía de independencia, si bien es una cuestión que radica en las características personales del consejero (Stein y Plaza, 2011), puede facilitarse si se articulan mecanismos que velen por ella. Si el papel del consejero independiente debe evolucionar hacia la representación de los intereses en el largo plazo, velando por un crecimiento medioambiental y socialmente sostenible y, como representantes últimos del capital flotante de la compañía, en nuestra opinión, el supervisor del mercado podría jugar un papel más activo en su designación.

Al fin y al cabo las entidades supervisoras del mercado (Comisión Nacional del Mercado de Valores en España o la Securities and Exchange Commission en EE.UU.) podrían garantizar un adecuado nivel de competencia. El modelo podría alcanzar diversos grados de intervención en el nombramiento de los consejeros independientes. Desde el más suave en el que el supervisor de los mercados se podría limitar a la creación de un registro de personas capacitadas para desempeñar la figura de consejero independiente, dejando el nombramiento a la empresa, a otro en el que el propio supervisor nombrase alguno o todos los independientes, lo que sin duda dotaría de mayor diversidad al debate en los órganos de gobierno de las entidades, en línea con las demandas del Libro Verde del Gobierno corporativo (Comisión Europea, 201 la). En la versión más suave, la incorporación al registro debería considerar los años de experiencia en cargos de alta dirección, auditoría, 
conocimiento de los sectores que podría aportar. Por otra parte, la inscripción en el registro sería la puerta para establecer obligaciones de formación continuada en temas coyunturales de los consejeros.

Por otra parte, los consejeros independientes deberían tener la responsabilidad de informar a la Comisión sobre el cumplimiento de los objetivos sobre Responsabilidad Social de la compañía, siendo responsables de dicha información y de la existencia de un tono de control adecuado sobre los activos que la sociedad civil pone a disposición de la compañía.

Si bien este modelo sigue siendo un modelo de mercado, frente a otro más intervencionista en el que el supervisor del mercado nombrase una parte o todos los consejeros independientes, consideramos que podría ser un avance en la creación de un clima de gobierno eficaz y socialmente responsable y respetuoso con el medioambiente.

\section{Conclusiones}

La crisis financiera iniciada en 2007 ha revelado algunas debilidades de las instituciones capitalistas que requieren ser revisadas. En nuestra opinión, muchos de los fallos a abordar residen no tanto en la regulación existente sino en la aplicación efectiva de los mecanismos y, especialmente, en una vigencia de los principios que subyacen en la misma, más allá de la letra de la norma que los regula. Por este motivo, en el trabajo abogamos por una regulación del gobierno de las entidades fundamentada en principios, combinado con órganos de supervisión del mercado, encargados de aportarle confianza, en lugar de una detallada legislación que burocratizaría los esquemas de gobierno y que, a la postre, podría convertirse en un mero listado de cumplimiento, sin reflejo en un mejor control de los riesgos de las entidades.

Por otra parte, paulatinamente, los reguladores, especialmente el europeo, están incorporando los intereses de otros "stakeholders" en el círculo a proteger con su actividad reguladora, yendo más allá de la pura visión del accionista, por lo que además del marco explicativo que ofrece la teoría de la agencia es preciso incorporar un nuevo contrato, adicional al existente entre gestor y propietario, que vincula a la entidad con la sociedad que le cede el uso de bienes públicos que deben ser protegidos. En definitiva, las entidades no sólo gestionan los recursos que controlan en exclusiva, sino que también cogestionan con el resto de la Huma- 
nidad una serie de bienes públicos. El disfrute de esos bienes públicos no debería suponer la privación de los mismos para terceros o para generaciones futuras. De esta manera el concepto de riesgo debe ir más allá del concepto de riesgo de la entidad y trasciende de los que exclusivamente conciernen a los propietarios de la misma. La realización de actividades tiene impacto en el medioambiente y el gobierno de las sociedades debería incorporar una visión respetuosa con estos recursos.

El modelo institucional de los mecanismos de control es mixto: por un lado, existen instituciones que se regulan por mecanismos de mercado como la auditoría de la información financiera o la calificación crediticia, en las que el régimen de responsabilidad es la principal salvaguarda, de carácter disuasorio, ante comportamientos oportunistas. Por otro, existen instituciones de carácter público encargadas de velar por el mercado de valores, por las entidades financieras o las compañías de seguros, que en alguno de los casos gozan de equipos de investigación que se instalan en las entidades $y$, en paralelo a los mecanismos de mercado, vigilan el estado financiero de las mismas; sin embargo, no por ello han podido evitar algunos casos de intervenciones bancarias, por ejemplo en España.

Por tanto, nuestra propuesta trata de incidir en el funcionamiento eficaz de las instituciones de acuerdo a los principios que las rigen y no a la letra de la norma que en cada momento las regula; por ese motivo, nuestra propuesta pone el acento en la creación de entornos favorables al control de riesgos desde el gobierno de las entidades. Para hacer eficaz dicha propuesta, y ésta es la principal aportación que realizamos en este trabajo frente a la literatura previa, se trataría de revitalizar la figura del consejero independiente para que pase de ser un representante de los accionistas minoritarios a ser una figura que encarne los intereses de los "stakeholders" en el máximo órgano de gobierno de las entidades, con unos niveles de competencia verificables por el órgano responsable de proteger el mercado y que esté obligado a rendir cuentas sobre el desempeño de la entidad en cuanto a su responsabilidad social. 


\section{Bibliografía}

AKERLOF, G.A. (1970). "The market for lemons: quality uncertainty and the market mechanism", The Quarterly Journal of Economics, Vol 84 (3), pp. 488-500

ARGANDOÑA, A. (1998): "La teoría de los stakeholders y el bien común". Documento de investigación n ${ }^{\circ}$ 355. Edita IESE. Disponible en: http://www.iese.edu/research/ pdfs/DI-0355.pdf

Balcaen, S. y OOGHE, H. (2006): "35 years of studies on business failure: an overview of the classic statistical methodologies and their related problems", The British Accounting Review, 38, pp. 63-93.

Comisión Especial para el Estudio de un Código ético de los Consejos de AdministraCIÓN DE LAS SOCIEDADES. Código Olivencia (1998): El Gobierno de las Sociedades Cotizadas. Madrid

Comisión EUROPEA (2010a): Comission Staff Working Document. Corporate Governance in Financial Institutions: Lessons to be drawn from the current financial crisis, bestpractices. Bruselas. Disponible en: http://ec.europa.eu/internal market/ company/docs/modern/sec2010_669_en.pdf

- (2010b): Libro Verde. Política de auditoría: Lecciones de la crisis. Bruselas. http://ec.europa.eu/internal market/consultations/docs/2010/audit/ green paper audit es.pdf

- (201 la): Libro Verde. La normativa de gobierno corporativo de la UE. Bruselas. http://ec.europa.eu/internal market/company/docs/modern/ com2011-164 es.pdf\#page $=2$

- (2011 b): Communications from the Commission to the European parliament, the European Economic and Social Committee and the Committee of the Regions. Social Business Initiative. Creating a favourable climate for social enterprises, key stakeholders in the social economy and innovation. Bruselas. Disponible en: $\underline{\text { http:// }}$ eur-lex.europa.eu/LexUriServ/LexUriServ.do?uri=COM:2011:0682:FIN:EN:PDF

- (201 1c): Propuesta de Reglamento del Parlamento Europeo y del Consejo por el que se modifica el Reglamento (CE) n 1060/2009 sobre las agencias de calificación crediticia. Bruselas. Disponible en: http://ec.europa.eu/internal market/ securities/docs/agencies/COM 2011747 en.pdf 
- (2011d). Propuesta de Reglamento del Parlamento Europeo y del Consejo sobre los requisitos específicos para la auditoría legal de las entidades de interés público. Bruselas. Disponible en: http://ec.europa.eu/internal market/auditing/ docs/reform/regulation es.pdf

Comt, A. (1980): El Capitalismo, Barcelona, Oikos Tau.

GalBraith, J. K. (1987): Historia de la Economía, Barcelona, Ariel.

G-20 (2009a): The Global Plan for Recovery and Reform, 2 april. Disponible en: http://www.g20.org/images/stories/docs/eng/london.pdf

- (2009b): Leaders' Statement: The Pittsburg Summit, 24-25 sept. Disponible en: http://www.g20.org/images/stories/docs/eng/pittsburgh.pdf

- (2010): Leaders' Declaration. Seoul Summit, 11-12 nov. Disponible en: http:// www.g20.org/images/stories/docs/eng/seoul.pdf

InTERNATIONAL FEDERATION OF ACCOUNTANTS (2012): Investor demand for environmental, social and governance disclosures. Implications for professional Accountants in Business. Ed. IFAC. Disponible en: http://www.ifac.org/publications-resources/ investor-demand-environmental-social-and-governance-disclosures

JENSEN, M.C. Y MECKLING, W.H. (1976): "Theory of the Firm: managerial behavior, agency costs and ownership structure". Journal of Financial Economics, 3(4), pp. 305-360

MADDISON, A. (1986): Las fases del desarrollo capitalista. Una historia económica cuantitativa, México, Fondo de Cultura Económica.

SIKKA, P. (2009): "Financial Crisis and the salience of the auditors". Accounting, Organizations and Society, 34, pp. 868-873.

Stein, G. y PlazA, S. (2011): "El papel del consejero independiente en la supervisión y rotación del CEO". IESE Business School, Estudio-133. Barcelona.

TwEEDIE, D. (2010): Prepared Statement to the meeting of the COUNCIL OF THE EUROPEANUNION (Economic and Financial Affairs). 16 March 2010. Disponible en: hitp://www.ifrs.org/News/Announcements+and+Speeches/ChairmanAddressesECOFIN.htm 